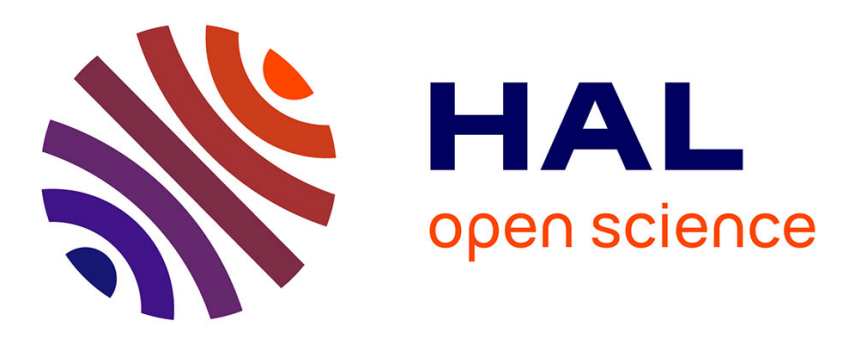

\title{
Subcritical Hopf bifurcation in dynamical systems described by a scalar nonlinear delay differential equation
}

\author{
L. Larger, Jean-Pierre Goedgebuer, T. Erneux
}

\section{- To cite this version:}

L. Larger, Jean-Pierre Goedgebuer, T. Erneux. Subcritical Hopf bifurcation in dynamical systems described by a scalar nonlinear delay differential equation. Physical Review E : Statistical, Nonlinear, and Soft Matter Physics, 2004, 69 (3), pp.036210. 10.1103/PhysRevE.69.036210 . hal-00096987

\section{HAL Id: hal-00096987 \\ https://hal.science/hal-00096987}

Submitted on 17 May 2021

HAL is a multi-disciplinary open access archive for the deposit and dissemination of scientific research documents, whether they are published or not. The documents may come from teaching and research institutions in France or abroad, or from public or private research centers.
L'archive ouverte pluridisciplinaire HAL, est destinée au dépôt et à la diffusion de documents scientifiques de niveau recherche, publiés ou non, émanant des établissements d'enseignement et de recherche français ou étrangers, des laboratoires publics ou privés. 


\title{
Subcritical Hopf bifurcation in dynamical systems described by a scalar nonlinear delay differential equation
}

\author{
Laurent Larger* and Jean-Pierre Goedgebuer* \\ Laboratoire d'Optique P. M. Duffieux, CNRS UMR No. 6603, Université de Franche-Comté, Route de Gray, \\ 25030 Besançon Cedex, France
}

Thomas Erneux

Groupe d'Optique Non Linéaire Théorique, Université Libre de Bruxelles, Campus Plaine, Case Postale 231, 1050 Bruxelles, Belgium

(Received 10 July 2003; published 31 March 2004)

\begin{abstract}
A subcritical Hopf bifurcation in a dynamical system modeled by a scalar nonlinear delay differential equation is studied theoretically and experimentally. The subcritical Hopf bifurcation leads to a significant domain of bistability where stable steady and time-periodic states coexist.
\end{abstract}

DOI: 10.1103/PhysRevE.69.036210

\section{INTRODUCTION}

Delay differential equations (DDEs) have attracted a lot of attention in the field of nonlinear dynamics. They are used to model complex phenomena like physiological diseases [1], population dynamics where a finite maturation time is needed [2], and neuronal networks [3]. DDEs also describe the response of nonlinear optical devices, such as Ikeda optical ring cavities [4], external cavity semiconductor lasers [5], or optoelectronic oscillators [6-8]. Many of these systems are accurately described by a single scalar DDE of the form

$$
\varepsilon y^{\prime}=-y+f(\lambda, y(t-1)),
$$

where $f(\lambda, y)$ denotes a nonlinear function of $y, \lambda$ is a control or bifurcation parameter, and $\varepsilon$ corresponds to the decay time of $y$ normalized by the delay. Provided $\varepsilon$ is sufficiently small, the response of the system can be very rich ranging from periodic to high dimensional chaotic outputs.

The complexity and the diversity of dynamical regimes described by Eq. (1) is well documented in the limit of large delays (limit $\varepsilon$ small). These regimes were studied by Losson et al. [9] who explored the coexisting periodic solutions and the fractal structure of their basin of attraction in the case of an analytically integrable piecewise constant function $f()$. For lasers subject to delay $[11,12]$, it was shown that these coexisting periodic solutions result from isolated branches of solutions that accumulate as the delay increases. The application to dynamical memory storage was studied numerically and experimentally by Foss et al. [10] and by Aida et al. [13]; in the latter case, the nonlinear function $f()$ was a cosine corresponding to the case of an electrooptic experimental setup in which a tunable two-wave interference figure is involved. In all these setups, periodic solutions are determined irrespective to their amplitude.

In this paper, we concentrate on the first Hopf bifurcation which can be investigated by a local analysis. This bifurcation is well documented $[4,13,15,23]$, has been analyzed

\footnotetext{
*Also at GTL CNRS Metz, UMR 6603, 2-3 rue Marconi, 57000 Metz, France.
}

PACS number(s): 05.45.-a, 82.40.Bj, 02.30.Ks

mathematically for small $\varepsilon[16,17]$, and the supercritical transition to a stable limit-cycle (see Fig. 1, left) has been observed experimentally. On the other hand, the case of a subcritical bifurcation (see Fig. 1, right) is less studied for DDE problems although it appears in applications [14]. A subcritical Hopf bifurcation leads to a branch of unstable solutions but may fold back and exhibit stable periodic solutions coexisting with stable steady states. The main objective of this paper is to determine the size of this overlap which has not been addressed in previous mathematical studies [18]. As we shall demonstrate on a specific problem, the domain of coexistence can be determined analytically from Eq. (1) in the limit $\varepsilon$ small. By contrast to subcritical Hopf bifurcation problems found for two variable ordinary differential equations [19], this domain is substantially important. An elementary circuit is constructed in order to evaluate experimentally the validity of our theory.

In Ref. [20], the direction of the Hopf bifurcation is determined for both $f(\lambda, y)$ and $\varepsilon$ arbitrary by using the Lindstedt-Poincaré method $[21,22]$. In this paper, we determine the direction of bifurcation for the case $\varepsilon=0$ by using a different and simpler method.

The paper is organized as follows. In Sec. II, we describe the conditions for a subcritical bifurcation and show that there exist severe restrictions on $f($ ). Our analysis motivates the design of an experimental system which is described in Sec. III. Experimental and analytical bifurcation diagrams are then compared. Finally, we discuss our main results in Sec. IV.

\section{THE HOPF BIFURCATION}

The analysis of the Hopf bifurcation of Eq. (1) for a general nonlinear function $f(\lambda, y)$ is detailed in Ref. [20] using
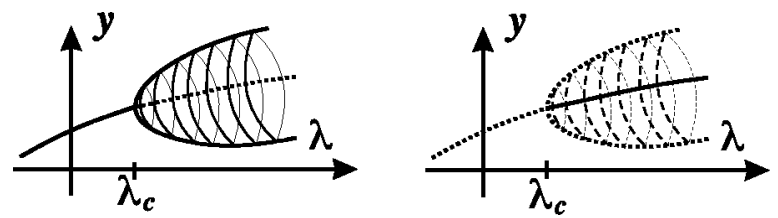

FIG. 1. Left: supercritical Hopf bifurcation. The branch of stable periodic solutions overlaps the branch of unstable steady states. Right: subcritical Hopf bifurcation. The branch of unstable periodic solutions overlaps the branch of stable steady states. 
the Lindstedt-Poincare perturbation method [21,22]. In this section, we concentrate on the case $\varepsilon=0$. Equation (1) then reduces to the map

$$
y_{n+1}=f\left(\lambda, y_{n}\right) \text {. }
$$

Assuming a basic solution $y_{s}(\lambda)$ satisfying

$$
y_{s}=f\left(\lambda, y_{s}\right) \text {, }
$$

we find from the linearized theory that a Hopf bifurcation point $\lambda=\lambda_{c}$ verifies the condition

$$
f_{y}\left(\lambda_{c}, y_{s}\left(\lambda_{c}\right)\right)=-1 \text {. }
$$

$f_{y}$ means the partial derivative of $f()$ with respect to $y$ evaluated at $\lambda=\lambda_{c}$. The stability of $y=y_{s}(\lambda)$ in the vicinity $\lambda=\lambda_{c}$ is investigated by determining the solution of the linearized problem for $\lambda=\lambda_{c}+\Lambda$ where $\Lambda$ is small. We find that the stability condition depends on the sign of $a$ defined by $[15]$

$$
a \equiv-\left(f_{y y} f_{\lambda}+2 f_{y \lambda}\right),
$$

where all partial derivatives are evaluated at $\lambda=\lambda_{c}$. The steady state solution is stable (unstable) if

$$
a \Lambda<0 \quad(a \Lambda>0) .
$$

We next wish to determine the direction of bifurcation, i.e., how the amplitude of the periodic solution changes as we deviate from the Hopf bifurcation point. Note that a periodic solution of Eq. (1) means a period 2 fixed point of the map (2) satisfying the two conditions

$$
\begin{aligned}
& y_{n+1}=f\left(\lambda, y_{n}\right), \\
& y_{n}=f\left(\lambda, y_{n+1}\right) .
\end{aligned}
$$

Introducing the deviations $u_{n}=y_{n}-y_{s}$ and $u_{n+1}=y_{n+1}$ $-y_{s}$ and expanding for small $u_{n}, u_{n+1}$, and $\Lambda=\lambda-\lambda_{c}$, we find

$$
\begin{gathered}
u_{n+1}=-u_{n}+\left(\frac{f_{\lambda} f_{y y}}{2}+f_{y \lambda}\right) \Lambda u_{n}+\frac{1}{2} f_{y y} u_{n}^{2}+\frac{1}{6} f_{y y y} u_{n}^{3}+\cdots, \\
u_{n}=-u_{n+1}+\left(\frac{f_{\lambda} f_{y y}}{2}+f_{y \lambda}\right) \Lambda u_{n+1} \\
+\frac{1}{2} f_{y y} u_{n+1}^{2}+\frac{1}{6} f_{y y y} u_{n+1}^{3}+\cdots
\end{gathered}
$$

Now assuming the scalings

$$
u_{n}=O(\delta), \quad u_{n+1}=O(\delta), \quad \text { and } \Lambda=O\left(\delta^{2}\right),
$$

we use Eq. (9) and eliminate $u_{n+1}$ in Eq. (10). Simplifying, we find

$$
0=a \Lambda u_{n}+b u_{n}^{3}
$$

where $a$ is defined by Eq. (5) and $b$ is given by

$$
b \equiv-\left(\frac{1}{2} f_{y y}^{2}+\frac{f_{y y y}}{3}\right) .
$$

Equation (12) then gives

$$
u_{n}=\sqrt{-\frac{a \Lambda}{b}}
$$

provided $a \Lambda / b<0$. A supercritical (subcritical) bifurcation means that the periodic solution overlaps an unstable steady state (stable steady state). Taking into account the inequalities (6), a supercritical (subcritical) bifurcation then implies the condition

$$
b<0(b>0) .
$$

Thus the sign of $b$ determines the direction of bifurcation. Because of the first term in Eq. (13), a necessary condition for a subcritical bifurcation where $b>0$ is that $f_{y y y}<0$ whatever the sign of $f_{y y}$. This underlines the essential role of the cubic correction term.

To further study the effect of the nonlinear correction terms, we introduce the third-order polynomial function given by

$$
f(\lambda, y)=y\left(A+B y+C y^{2}\right),
$$

where $A, B$, and $C$ are functions of $\lambda$. Our basic steady state solution is $y_{s}=0$ and for simplicity, we assume that the Hopf bifurcation point is located at $\left(\lambda_{c}, y_{c}\right)=(1,0)$. Using condition (1) and assuming that $a$ and $b$ defined by Eqs. (5) and (13) are both positive, we find the conditions

$$
A^{\prime}(1)=-1 \text { and } C(1)<-B^{2}(1) .
$$

The first condition is realized with $A=-\lambda$. The second condition is verified if $B=0$ and $C<0$. For many Hopf bifurcation problems in nonlinear optics, the bifurcation parameter is a feedback parameter and often appears as a multiplicative weighting factor in the nonlinear growth function. Choosing $C=-\alpha^{2} \lambda$, our function (16) becomes

$$
f(\lambda, y)=-\lambda y\left[1+(\alpha y)^{2}\right] .
$$

The next step is to take into account the unavoidable bounded character of the physical growth function $f(\lambda, y)$ as $y$ becomes large. This is, for example, the effect of saturation of the gain medium in an optical system. This can be modeled by dividing Eq. (18) by a fourth-order polynomial such as $\left[1+(\beta y)^{4}\right]$. The parameter $\beta$ controls the amount of saturation. Our function now is

$$
f(\lambda, y)=-\lambda \frac{y\left[1+(\alpha y)^{2}\right]}{1+(\beta y)^{4}} .
$$

For large delays ( $\varepsilon$ small), Eq. (1) reduces to the map (2). Period 1 and period 2 fixed points of this equation correspond to steady state and square-wave pulsating solutions of Eq. (1). In the case of Eq. (19), these fixed point solutions can be determined analytically (see the Appendix). We find 


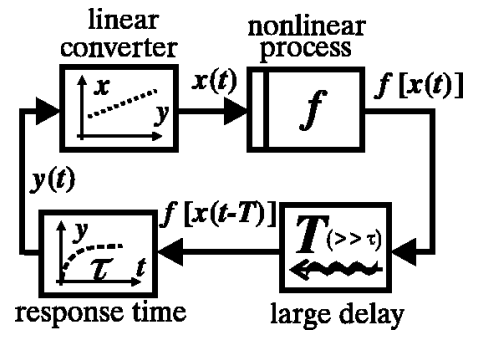

FIG. 2. Block diagram of a typical experiment described by a DDE.

that the period 2 solutions that emerge from $\lambda=1$ satisfy the condition $y_{n+1}=-y_{n}$ where $y_{n}(\lambda)$ satisfies the implicit solution

$$
\lambda=\frac{1+\left(\beta y_{n}\right)^{4}}{1+\left(\alpha y_{n}\right)^{2}}
$$

The bifurcation diagram $y_{n}(\lambda)$ is shown numerically in Fig. 5 for $\alpha=3$ and $\beta=2$. The location of the limit point where the subcritical Hopf bifurcation branch folds back can be determined from Eq. (20); see the Appendix. It is located at

$$
\lambda_{f}=2(\beta / \alpha)^{4}\left[\sqrt{(\alpha / \beta)^{4}+1}-1\right] .
$$

The domain of coexistence is defined by the interval $\lambda_{c}$ $-\lambda_{f}$. It is the largest in the limit $\beta \rightarrow 0\left(\lambda_{f} \rightarrow 0\right)$. Note that the saturation parameter $\beta$ cannot be zero. Without $\beta$, the Hopf bifurcation branch does not fold back. If $\beta=0, \lambda_{f}=0$ but $y_{n}(0)=\infty$ and the subcritical unstable Hopf branch is hyperbolic with a vertical asymptote at $\lambda=0$. In the following section, we realize an experimental system that is described by a DDE with a growth function close to Eq. (19).

\section{EXPERIMENTS}

The experimental setup for a physical oscillator ruled by a DDE is represented schematically in Fig. 2. It consists of a closed loop system formed by a linear tunable source, a nonlinear transformation $f()$, a delay time $T$, and a first-order low-pass filter. Such systems have been used previously in different problems in nonlinear optics $[8,15]$ and in electronics $[9,10,24]$. Here, we consider an electronic setup described in terms of a scalar DDE where the function $f$ will be designed in order to meet the conditions for a subcritical Hopf bifurcation.

The circuit is described in Fig. 3 and consists of the following.

(1) A voltage controlled oscillator (VCO), which corresponds to the linear block in the figure: it delivers an output sine wave form with an amplitude of $1 \mathrm{~V}$ and a frequency linearly related to the input tuning voltage through a tuning rate $S=25.4 \mathrm{kHz} / \mathrm{V}$; the central frequency (zero input) is adjusted to $\nu_{0}=36 \mathrm{kHz}$; the dynamical variable $y$ corresponds to the VCO output frequency $\nu$, or to its input tuning voltage.

(2) An electronic filter operating in the tuning range of the VCO: It is built using two resonant RLC second-order band-

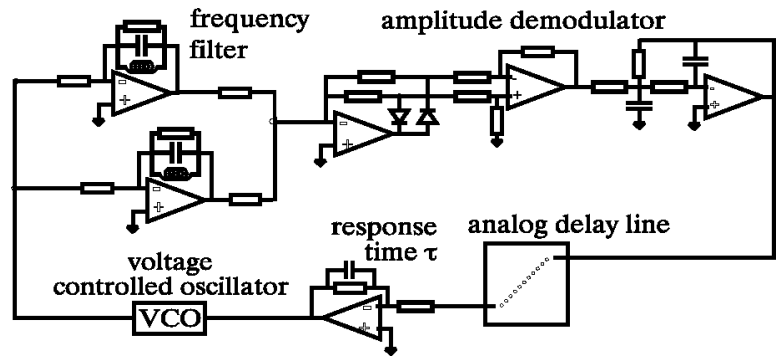

FIG. 3. Electronic circuit performing the experimental DDE.

pass filters, with resonance frequencies $\nu_{1}=80 \mathrm{kHz}$ and $\nu_{2}$ $=99 \mathrm{kHz}$, and quality factors $Q_{1}=2.12$ and $Q_{2}=3.1$, respectively. The two filters operate in parallel, their outputs are added with a relative factor $g_{2 / 1}=0.22$. To demodulate the resulting output amplitude, a full-wave rectifier and a low-pass filter with a cutoff frequency $\nu_{c}$ of $3 \mathrm{kHz}$ are used. This electronic filter followed by the amplitude demodulator performs the nonlinear function $f$ involved in the dynamical process. The role of the low pass is two-fold: it filters out the FM carrier frequency of the VCO output [see Eq. (22) and output trace in the left part of Fig. 4], and it defines the first-order dynamical process expressed in Eq. (1), with a time constant $\tau=1 / 2 \pi \nu_{c}=12 \mu \mathrm{s}$.

(3) An electronic delay line performed by an analog charge-coupled device memory of 256 samples: The resulting delay is $T=2.05 \mathrm{~ms}$, thus performing a large delay DDE since $\varepsilon=\tau / T \simeq 0.0058$.

(4) A linearly controlled voltage amplifier is used to tune electronically the oscillator loop gain, which corresponds to the bifurcation parameter $\lambda$.

Following our description, the nonlinear oscillator is then modeled by Eq. (1), with the following nonlinear function:

$$
\begin{aligned}
f(\lambda, \nu)= & \lambda \mid \frac{i \nu / \nu_{1}}{1-\left(\nu / \nu_{1}\right)^{2}+i\left(\nu / \nu_{1}\right) /\left(\sqrt{2} Q_{1}\right)} \\
& +\frac{i g_{2 / 1}\left(\nu / \nu_{2}\right)}{1-\left(\nu / \nu_{2}\right)^{2}+i\left(\nu / \nu_{2}\right) /\left(\sqrt{2} Q_{2}\right)} \mid
\end{aligned}
$$

In order to best fit the nonlinear function profile with the shape (19) proposed theoretically, we adjusted the relative resonance frequencies of the two RLC filters, their quality factor, and relative amplitudes (parameters $\nu_{2}, Q_{2}$, and $g_{2 / 1}$ ). To match the experimental nonlinear function with the theoretical profile in the vicinity of the Hopf bifurcation point, we stored the latter in an arbitrary wave form generator, and fitted adequately the experimental parameters while scanning linearly in time the VCO output frequency in the open loop oscillator configuration. Figure 4 (left) represents the experimental plot of the optimized nonlinear function shape. Then, the feedback loop gain $\lambda$ and the operating point of the $\operatorname{VCO}\left(\nu_{0}\right)$ were tuned to a value near the Hopf point.

The bifurcation diagram in Fig. 4 (right) was then ob- 


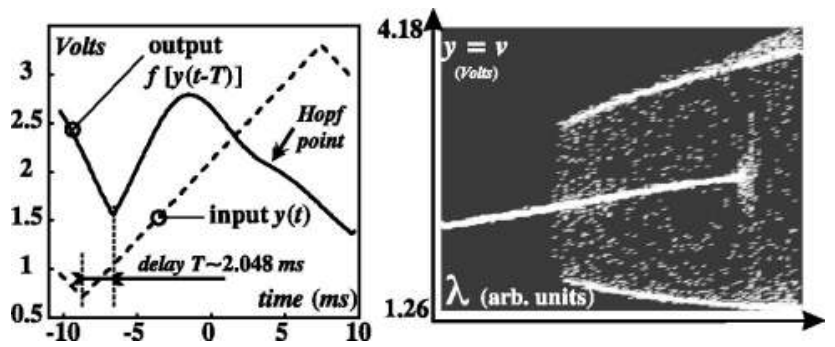

FIG. 4. Experimental bifurcation diagram. Left: input/output trace in the open loop configuration showing the nonlinear function and the delay when scanning linearly in time the VCO frequency (proportional to $y$ ). Right: the bifurcation diagram obtained by increasing or decreasing the bifurcation parameter in the vicinity of the Hopf bifurcation point.

tained in the closed loop operation, as the feedback gain was scanned back and forth around the Hopf value. The horizontal axis in Fig. 4 (right) is the voltage applied to the tuning feedback loop gain (proportional to the bifurcation parameter $\lambda$ ), and the vertical axis is the voltage at the VCO input (this voltage is proportional to the electronic frequency $\nu$, i.e., the dynamical variable $y$ ). The traces in Fig. 4 (right) have a brightness related to the amplitude probability for a given dynamical regime. When a steady state is the stable solution for a given bifurcation parameter value, i.e., for a given position along the horizontal axis, a single spot is seen on the vertical line on the oscilloscope screen (left part of the bifurcation diagram). When a cycle is the stable solution, for example, when the bifurcation parameter is above the Hopf bifurcation point, the oscillation is nearly a symmetric square wave form. The two bright spots observed for that regime on a vertical line correspond to the high and low levels of the square wave form (right part of the bifurcation diagram). Since the wave form is continuous in time, some diffuse spots are seen between the two plateaus, which correspond to the switching events between the levels of the square wave form. When three bright spots are seen on a vertical line (central part in the bifurcation diagram), it corresponds to the hysteresis region, where the steady state is visited when increasing the bifurcation parameter, while the cycle is visited for decreasing bifurcation parameter values. Note the white spots near the bifurcation point which indicate the typical slow time scale of the transient evolution near Hopf bifurcation point.

The bifurcation diagram can be compared with the diagram obtained numerically (see Fig. 5) when integrating the continuous time dynamics of Eq. (1). The function $f()$ used in the numerical simulation is modeled by Eq. (22), in which the parameters values are those measured in the experimental setup and given at the beginning of this section.

\section{DISCUSSION}

We have studied experimentally a subcritical Hopf bifurcation in a time delayed feedback system that is described by Eq. (1). The nonlinear growth function was designed after
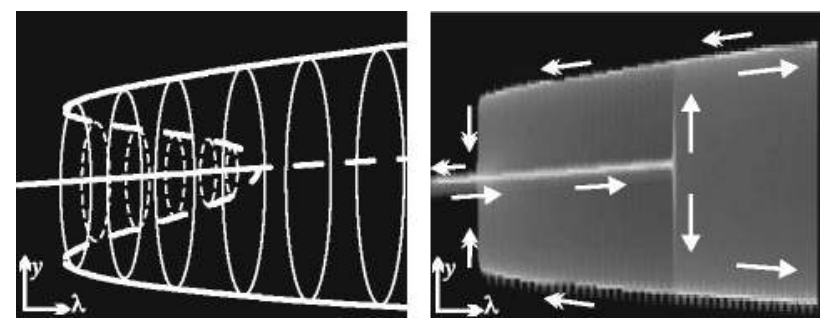

FIG. 5. Numerical bifurcation diagram. Left: Period 2 fixed points of the map 2 showing both the stable and unstable solutions. Right: stable steady and periodic solutions of the DDE (1).

determining theoretically conditions for a subcritical bifurcation. The experimental bifurcation diagram shows a significant domain of bistability between a stable limit cycle and a stable steady state. Note that the subcritical nature of the Hopf bifurcation is related to the existence of an unstable limit cycle near the Hopf bifurcation point. It is not possible to obtain experimentally a direct proof of the presence of the unstable cycle. That unstable cycle is anticipated through the observation of the hysteresis in the bifurcation diagram and by comparing with the analytical bifurcation diagram. However, such a hysteresis might also exist with a stable cycle which is not connected to the unstable branch born at the Hopf point. To test this, we tuned slowly and continuously the relative gain $g_{2 / 1}$ while scanning back and forth the bifurcation diagram. Doing so it was possible to diminish down to zero both the vertical and the horizontal extent of the hysteresis, thus indicating that the stable cycle was effectively connected to the unstable cycle.

Theoretically, we concentrated on the case of a subcritical Hopf bifurcation occurring in the DDE (1) and exhibiting a large delay ( $\varepsilon$ small). We have found that the domain of bistability is independent of $\varepsilon$ and that it can be significantly large. This contrasts to relaxation oscillators described by two first-order nonlinear ordinary differential equations [19].

\section{ACKNOWLEDGMENTS}

We acknowledge the EEC for its financial support in the frame of the OCCULT project (Grant No. IST-2000-29683). The research of T.E. was supported by U.S. Air Force Office of Scientific Research Grant No. AFOSR F49620-98-1-0400, National Science Foundation Grant No. DMS-9973203, the Fonds National de la Recherche Scientifique (Belgium), and the InterUniversity Attraction Pole program of the Belgian government.

\section{APPENDIX: LIMIT POINT OF PERIODIC SOLUTIONS}

We wish to determine the critical point $\lambda=\lambda_{f}$ where the stable limit cycle connects the unstable limit cycle appeared at the Hopf bifurcation point. To this end, we first note that the nonlinear function shown in Eq. (19) is an odd function of $y_{n}$. This implies that any period 2 fixed point corresponding to a cycle satisfies the equation $f(\lambda, y)=-y$ with $y \neq 0$, or equivalently, using the function in Eq. (19) the equation 


$$
\lambda\left[1+(\alpha y)^{2}\right]=1+(\beta y)^{4} .
$$

This equation admits two real solutions for $y^{2}$ if $\lambda_{f}<\lambda$ $<\lambda_{c}$. The condition for a double real root determines $\lambda$ $=\lambda_{f}$. This implies that the discriminant $\Delta=\lambda^{2} \alpha^{4}-4(1$ $-\lambda) \beta^{4}$ is zero. This equation admits a positive solution given by

$$
\lambda_{f}=2(\beta / \alpha)^{4}\left[\sqrt{(\alpha / \beta)^{4}+1}-1\right] .
$$

[1] M.C. Mackey and L. Glass, Science 197, 287 (1977).

[2] R.M. May, Nature (London) 261, 459 (1976).

[3] J. Wei and S. Ruan, Physica D 130, 255 (1999).

[4] K. Ikeda, Opt. Commun. 30, 257 (1979).

[5] R. Lang and K. Kobayashi, IEEE J. Quantum Electron. 16, 347 (1980)

[6] H.M. Gibbs, F.A. Hopf, D.L. Kaplan, and R.L. Schoemacker, Phys. Rev. Lett. 46, 474 (1981).

[7] F. Arecchi, W. Gadomski, and R. Meucci, Phys. Rev. A 34, 1617 (1986).

[8] L. Larger, J.-P. Goedgebuer, and J.M. Mérolla, IEEE J. Quantum Electron. 34, 594 (1988).

[9] J. Losson, M.C. Mackey, and A. Longtin, Chaos 3, 167 (1993).

[10] J. Foss, F. Moss, and J. Milton, Phys. Rev. E 55, 4536 (1997).

[11] P.M. Alsing, V. Kovanis, A. Gavrielides, and T. Erneux, Phys. Rev. A 53, 4429 (1996).

[12] D. Pieroux, T. Erneux, A. Gavrielides, and V. Kovanis, SIAM (Soc. Ind. Appl. Math.) J. Appl. Math. 61, 966 (2000).

[13] T. Aida and P. Davis, IEEE J. Quantum Electron. 28, 686 (1992).

[14] T. Kalmar-Nagy, G. Stepan, and F.C. Moon, Nonlinear Dyn. 26, 121 (2001).
[15] L. Larger, M.W. Lee, J.P. Goedgebuer, T. Erneux, and W. Elflein, J. Opt. Soc. Am. B 18, 1063 (2001).

[16] S.-N. Chow and J. Mallet-Paret, in Coupled Nonlinear Oscillators, edited by J. Chandra and A.C. Scott (North-Holland, Amsterdam, 1983), p. 7.

[17] S.N. Chow, J.K. Hale, and W. Huang, Proc. R. Soc. Edinburgh, Sect. A: Math. 120, 223 (1992).

[18] J.K. Hale and W. Huang, J. Diff. Eqns. 114, 1 (1994).

[19] S.M. Baer and T. Erneux, SIAM (Soc. Ind. Appl. Math.) J. Appl. Math. 46, 721 (1986); 52, 1651 (1992).

[20] T. Erneux, L. Larger, M.W. Lee, and J.-P. Goedgebuer, Physica D (to be published).

[21] P.G. Drazin, Nonlinear Systems (Cambridge University Press, Cambridge, 1992).

[22] A. Nayfeh, Perturbation Methods (Wiley Interscience, New York, 1973).

[23] G. Iooss and D.D. Joseph, Elementary Stability and Bifurcation Theory, Und. Texts in Mathematics (Springer-Verlag, New York, 1980); 2nd ed. (Springer, New York, 1990).

[24] L. Larger, V.S. Udaltsov, and J.P. Goedgebuer, Electron. Lett. 36, 199 (2000). 